\title{
Artificial Neural Network Modeling of an Inverse Fluidized Bed Bioreactor
}

\author{
*RAJASIMMAN, M, GOVINDARAJAN, L, KARTHIKEYAN, C
}

\author{
Department of Chemical Engineering, Annamalai Unive, rsity \\ Annamalai Nagar-608 002, Tamil Nadu, India.
}

\begin{abstract}
The application of neural networks to model a laboratory scale inverse fluidized bed reactor has been studied. A Radial Basis Function neural network has been successfully employed for the modeling of the inverse fluidized bed reactor. In the proposed model, the trained neural network represents the kinetics of biological decomposition of pollutants in the reactor. The neural network has been trained with experimental data obtained from an inverse fluidized bed reactor treating the starch industry wastewater. Experiments were carried out at various initial substrate concentrations of 2250, 4475, 6730 and $8910 \mathrm{mg} \mathrm{COD} / \mathrm{L}$ and at different hydraulic retention times $(40,32,24,26$ and $8 \mathrm{~h})$. It is found that neural network based model has been useful in predicting the system parameters with desired accuracy. @JASEM
\end{abstract}

Artificial Neural Networks (ANN) have been established as a tool for effortless computation and its application in environmental engineering field is very promising and has gained extensive interest (Hamoda et al., 1999, Hack and Kohne, 1996, Gontarski et al., (2000), Bongards, 2001, Hamed et al., 2004). ANN have been successfully employed in solving problems in areas such as fault diagnosis, process identification, property estimation, data smoothing and error filtering, product design and development, optimization, dynamic modeling and control of chemical processes, for the prediction of vapor-liquid equilibrium (VLE) data and estimation of activity coefficients. The purpose of using artificial neural networks in wastewater treatment system is to reduce the number of experiments that are being carried out to characterize the system.

ANN has remarkable ability to derive meaningful information from complicated or imprecise data. It can be used to extract patterns and detect trends, which are too complex to be noticed by other computational technique (Mehrotra et al., 1997). Neural networks, inspired by the information processing strategies of the human brain, are proving to be useful in a variety of engineering applications. ANN may be viewed as paralleled computing tools comprising of highly organized processing elements called neurons which control the entire processing system by developing association between objects in response to their environment. The researches have proposed many architectures of the network .Two widely used network for modeling the non-linear problems in engineering systems are the Backpropagation and Radial Basis Function (RBF) networks.

Radial basis networks require lesser neurons than the standard feed forward back propagation networks and they can be trained in a fraction of time (Govindarajan, 2002). In this work, radial basis network function has been successfully incorporated for the prediction of degradation of organic matter present in starch wastewater treated in an inverse fluidized bed reactor. The proposed technique of using radial basis function requires only limited experimental values to predict the behaviour of the system. A simple well-trained neural network can be employed to overcome the modelling problems of reactor without prior knowledge of the relationships of process variables under investigation.

Radial Basis Function Network

Radial basis function networks form one of the essential categories of neural networks. A RBF network is a two-layer network whose output units form a linear combination of the basis functions computed by the hidden units. A function is radially symmetric if its output depends on the distance of the input sample from another stored vector. Neural networks whose node functions are radially symmetric functions are referred to as Radial Basis Function Nets.

The transfer function for a radial basis neuron is radbas. The radial basis neuron receives as net input the vector distance between its weight vector $\mathrm{w}$ and the input vector $p$, multiplied by the bias $b$. The basis functions in the hidden layer produce a localized response to the inputs i.e each hidden unit has a localized receptive field. The basis function can be viewed as the activation function in the hidden layer. The outputs of the hidden unit lie between 0 and 1 . The closer the input to centre of the Gaussian, the larger the response of the node. The node produces an identical output for inputs with equal distance from the center of the Gaussian; it is called a radial basis. The output unit form a linear combination of the 
nonlinear basis functions and thus the over all network performs a nonlinear transformation of the input.

\section{RBF Training Procedure}

The radial basis neural networks have been designed by the using the function newrb available in the neural network toolbox supported by MATLAB 7.0. The function newrb iteratively creates a radial basis network by including one neuron at a time. Neurons are added to the network until the sum squared error is found to be very small or the maximum numbers of neurons are reached. At each iteration the input vector, which will result in lowering the network error most, is used to create a radial basis neuron.

During the training, each of the connecting weights of the individual neuron is compared with input signals. The distance between the connecting weights determines the output of hidden neurons and input vector, which is further, multiplied by bias an additional scalar quantity being added between neuron and fictitious neuron. The output is propagated in a feed forward direction to output layer neuron, which will give output if the connection weights are close to input signal. This output is compared with target vector. If the error reaches the error goal then training is completed otherwise the next neuron will be added.
The connecting weights are modified each time by changing maximum neurons and spread constant. The value of maximum neuron and spread constant are keep on changing till the network is trained properly. Radial basis networks can be used to approximate functions. newrb adds neurons to the hidden layer of a radial basis network until it meets the specified mean squared error goal.

The advantages of RBF are, the time taken in designing a radial basis network is often less when compared to the training a sigmoid / linear networks and the number of neurons required for designing the network is considerably less when compared to standard back propagation network (Govindarajan, 2005). The following steps are repeated until the network's mean squared error falls below goal as given in Fig.

i. The network is simulated

ii The input vector with the greatest error is found iii A radbas neuron is added with weights equal to that vector

iv The purelin layer weights are redesigned to minimize error

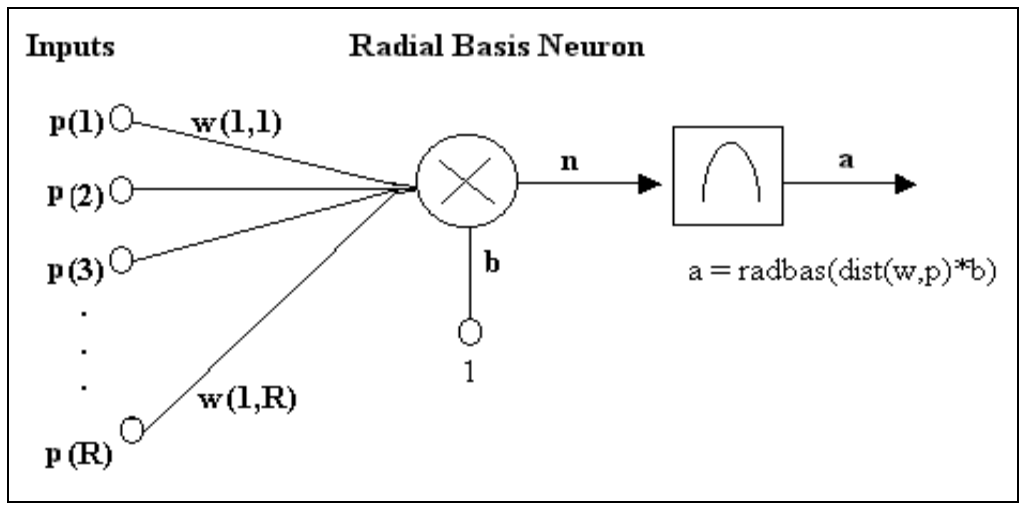

Fig. 1 Radial Basis Function Neuron Model

\section{MATERIALS AND METHODS}

Degradation of starch wastewater in Inverse Fluidized Bed Bioreactor (IFBBR) was carried out continuously in different stages by varying initial substrate concentration $(2250 \mathrm{mg} \mathrm{COD} / \mathrm{L}, 4475 \mathrm{mg} \mathrm{COD} / \mathrm{L}$, $6730 \mathrm{mg} \mathrm{COD} / \mathrm{L}$ and $8910 \mathrm{mg}$ COD/L) and hydraulic retention time $(40,32,24,16$ and $8 \mathrm{~h})$. Various experimental runs were conducted to provide information of the process behavior and to train the network. The particles were introduced into the reactor along with the substrate and inoculum. The air flow rate was adjusted according to bed height, for biomass growth. The growth of the biofilm on the surface of the particles was monitored at regular intervals. The reactor inoculation and startup procedure were followed according to Rajasimman and Karthikeyan, 2006.

Continuous degradation in IFBBR was started with an initial concentration of $2250 \mathrm{mg} \mathrm{COD} / \mathrm{L}$ and a HRT of $40 \mathrm{~h}$. The reactor was monitored continuously by measuring effluent COD values. When the reactor reaches steady state, the HRT was reduced to $32 \mathrm{~h}$ and reduction in COD was monitored. Experiment was 
continued with various HRT $(24,16$ and $8 \mathrm{~h})$. The performance of the reactor was studied based on COD removal efficiency. Experiments were repeated for various initial substrate concentrations at five different HRT by maintaining an optimum bed height $(80 \mathrm{~cm})$ and air flow rate $(62.50 \mathrm{cc} / \mathrm{s})$. The $\mathrm{pH}$ of the influent to the reactor was maintained at 6.0. The analyses were made according to the methods given in standard methods of analysis by American Public Health Association, APHA (1992). The experimental data were fed to the neural network to model the IFBBR in degrading the starch wastewater.

\section{RESULTS AND DISCUSSION}

The Radial Basis Function network was trained to predict the performance of IFBBR. The criterion used to evaluate the performance of the reactor is to determine the reduction in organic matter present in the starch industry wastewater. The neural network was trained with the influent substrate concentration, hydraulic retention time and effluent concentration of the reactor. The data used for the training at various hydraulic retention times of 40,32, 24, 16 and $8 \mathrm{~h}$ and at different initial substrate concentrations of 2250 , 4475, 6730 and $8910 \mathrm{mg} \mathrm{COD/L}$.

The neural network predicted data were compared with the experimental findings at the intermediate hours viz 36, 60, 84, 108, 132, 156, 180, 204, 228 hours of operation and at different initial substrate concentrations of 2250, 4475 and $6730 \mathrm{mg} \mathrm{COD} / \mathrm{L}$. For the initial substrate concentration $8910 \mathrm{mg}$ $\mathrm{COD} / \mathrm{L}$, the data were predicted for $48,96,144,192$, $240,288,336$ and $384 \mathrm{~h}$. The performance of the network was evaluated on the basis of an overall absolute error and root mean square error (RMSE) specified by the difference in the desired and actual outputs. A comparison of experimental values and the ANN predicted results were depicted in Figures 2 to 5. From these Figures it has been observed that artificial neural network modeling of system-based parameters are found to match exactly with the experimental data at various operating conditions of the reactor. The use of neural network for the prediction of the performance of IFBBR has been found to be valid and robust, eliminating the need for the complex mathematical and computations involved in the modeling of the IFBBR performance.
The absolute standard deviation and percentage root mean square error, used in this study for the evaluation of neural network model, is defined as Absolute Standard Deviation (ABSD)

$$
A B S D=\frac{\sum \mid(N N \text { value }- \text { Experimental value }) \mid}{\text { number of data points }}
$$

Root Mean Square Error (RMSE)

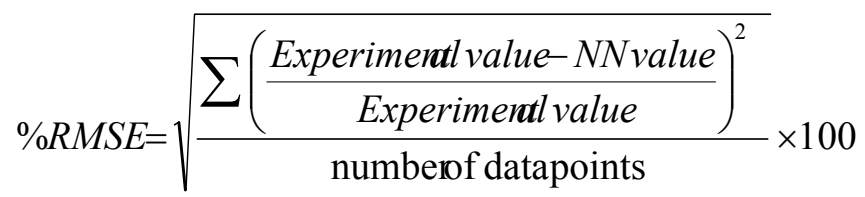

The Absolute Standard Deviation and percentage RMSE were found for each run and were tabulated in Table 1. From the Table it has been found that the deviations were well within the permissible limit. Thus artificial neural network modeling of IFBBR is highly justified for the treatment of starch industry wastewater.

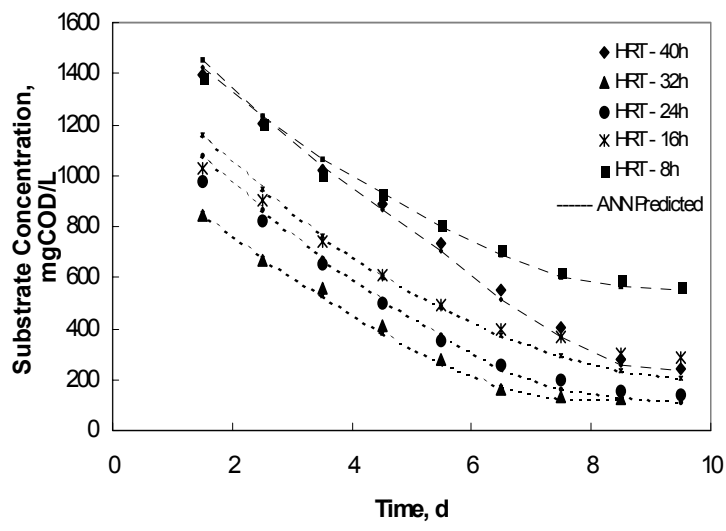

Fig. 2 Experimental and ANN Predicted COD Vs Time - Initial Substrate Concentration of $2250 \mathrm{mg} \mathrm{COD} / \mathrm{L}$ 


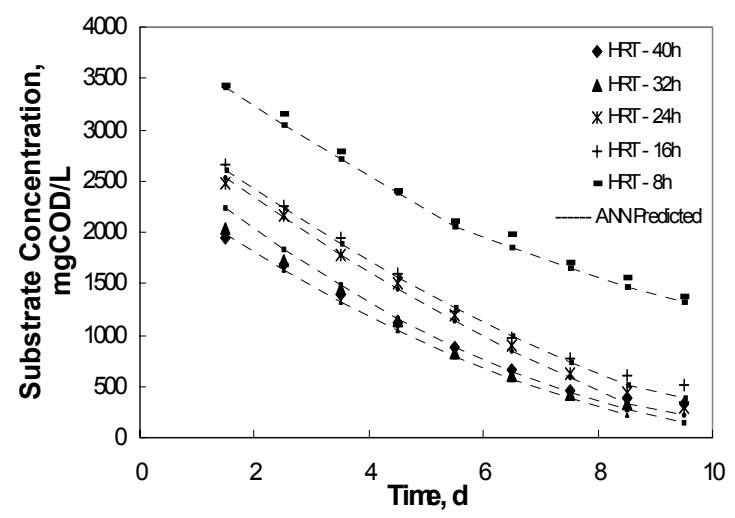

Fig. 3 Experimental and ANN Predicted COD Vs Time Initial Substrate Concentration of $4475 \mathrm{mg} \mathrm{COD} / \mathrm{L}$

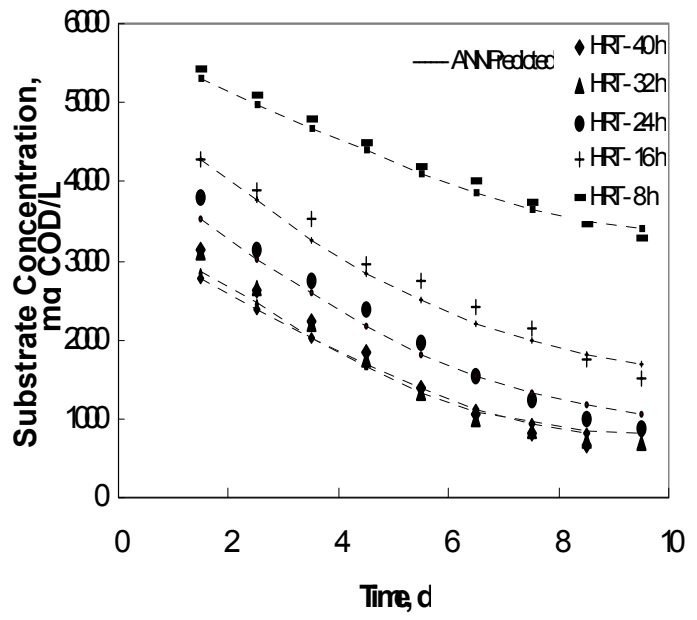

Fig. 4 Experimental and ANN Predicted COD Vs Time Initial Substrate Concentration of $6730 \mathrm{mg} \mathrm{COD} / \mathrm{L}$

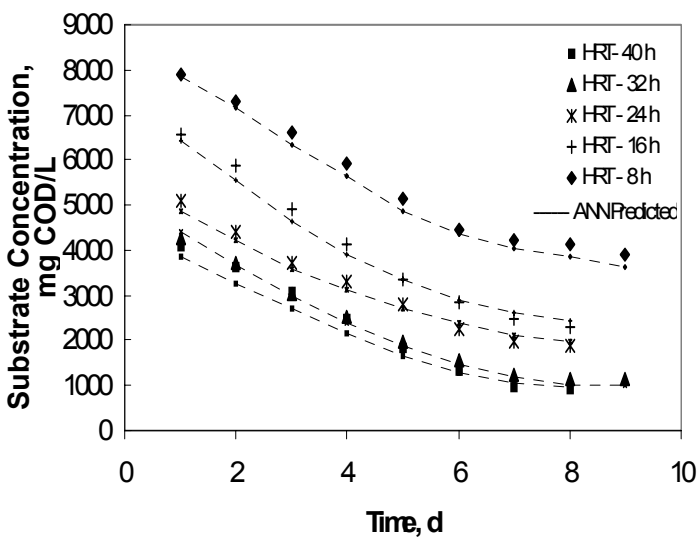

Fig. 5 Experimental and ANN Predicted COD Vs Time Initial Substrate Concentration of $8910 \mathrm{mg} \mathrm{COD} / \mathrm{L}$

Conclusion: In this study, the inverse fluidized bed bioreactor treating starch industry wastewater was modeled using artificial neural network. The ANN predicted values are compared with the experimental values and it is very close to the experimental values. The low RMSE values indicate the performance of ANN in predicting the system.

Acknowledgement: The authors wish to express their gratitude for the support extended by the authorities of Annamalai University, Annamalai Nagar, India in carrying out the research work in Environmental Engineering laboratory, Department of Chemical Engineering.

Table 1 Comparison between Experimental and Neural Network Predicated Values - Modeling of IFBBR Used for Treatment of starch Industry Effluent

\begin{tabular}{ccccccc}
\hline $\begin{array}{c}\text { Substrate } \\
\begin{array}{c}\text { Concentration, } \\
\text { mg COD/L }\end{array}\end{array}$ & HRT 40 h & HRT 32 h & HRT 24 h & HRT 16 h & HRT $8 \mathrm{~h}$ \\
\hline 2250 & ABSD & 25.56 & 10.33 & 34.11 & 48.33 & 58.78 \\
& \%RMSE & $5.32 \%$ & $3.20 \%$ & $11.54 \%$ & $14.41 \%$ & $7.98 \%$ \\
4475 & ABSD & 53.11 & 68.5 & 55.44 & 50.55 & 151.44 \\
& \%RMSE & $17.6 \%$ & $12.0 \%$ & $12.52 \%$ & $10.12 \%$ & $7.5 \%$ \\
6730 & ABSD & 79.6 & 86.5 & 95.6 & 123.2 & 168.6 \\
& \%RMSE & $10.6 \%$ & $7.1 \%$ & $5.81 \%$ & $6.1 \%$ & $5.65 \%$ \\
8910 & ABSD & 213 & 101.44 & 157.13 & 192 & 219 \\
& \%RMSE & $9.4 \%$ & $6.92 \%$ & $5.42 \%$ & $5.27 \%$ & $4.81 \%$ \\
\hline
\end{tabular}




\section{REFERENCE}

APHA, (1992). Standard methods for the examination of water and wastewater, $16^{\text {th }}$ edition, American Public Health Association, New York.

Bongards, M (2001). Improving the efficiency of a wastewater treatment plant by fuzzy control and neural networks. Water Science and Technology, 43 (11): 189 - 196.

Gontarski, C A; Rodrigues, P R; Mori, M; Prenem, L F (2000). Simulation of an industrial wastewater treatment plant using artificial neural networks. Computers \& Chemical Engineering, 24:1719-1723.

Govindarajan, L (2002). Radial Basis Neural Network Approach to VLE Data Predictions. M.E., Thesis, Annamalai University, Annamalai Nagar, India.

Govindarajan, L (2005). Optimal design of reactors. Ph.D Thesis, Annamalai University, Annamalai Nagar, India.
Hack, M; Kohne, M (1996). Estimation of wastewater process parameters using neural networks. Water Science and Technology, 33 (1): $101-115$.

Hamed, M M; Khalafallah, M G; Hassanien, E A (2004). Prediction of wastewater treatment plant performance using artificial neural networks. Environmental Modelling and Software, 19: 919 -928.

Hamoda, M F; Al-Ghusain, I A; Hassan, A H (1999). Integrated wastewater treatment plant performance evaluation using artificial neural networks. Water Science and Technology, 40 (7): 55-66.

Mehrotra, K; Mohan, C K; Ranka, S (1997). Elements of Artificial Neural Networks, Penram International, Mumbai.

Rajasimman, M; Karthikeyan, C (2006). Treatment of starch industry effluent in an inverse fluidized bed bioreactor," J. Appl Sci Environ Manage, 10 (1): $39-44$ 